Conclusion: Basically, this study shows that in patients with clinical indication of biological therapy by RA activity, a previous evaluation and a ultrasound are necessary in order to have a more accurate approach in making decisions; ultrasound is a practical, real-life, low-cost test to define activity in RA patients. Also we avoid high costs therapies for the management of rheumatoid arthritis, therefore we contribute not only to the health outcomes of patients but the health economic aspects in the management of RA.

Disclosure of Interests: Edwin Castillo: None declared, Michael Cabrera: None declared, Diana Buitrago-Garcia: None declared, Pedro Santos-Moreno Grant/research support from: Dr Santos has received research grants from Janssen, Abbvie and UCB, Speakers bureau: $\mathrm{Dr}$ Santos has received speaker fees from Sanofi, Lilly, Bristol, Pfizer, Abbvie, Janssen and UCB, Eva Cardozo: None declared, Ivania Ramirez: None declared, Danny Gomez: None declared, Sandra Farietta: None declared

DOI: 10.1136/annrheumdis-2019-eular.6473

\section{FRI0649 PREVALENCE OF ANCA AND ANA IN PATIENTS WITH PULMONARY TUBERCULOSIS}

AMAN SHARMA ${ }^{1}$, Yogesh Chander ${ }^{1,2}$, Manish Rathi $^{2}$, Gsrsnk Naidu ${ }^{1}$, Saket Jha ${ }^{1}$, Ranjana Minz ${ }^{3}$, Sanjay Jain ${ }^{1}$, Digambar Behera ${ }^{4}{ }^{1}$ Post Graduate Institute of Medical Education and Research, Chandigarh, Internal Medicine, Chandigarh, India; ${ }^{2}$ Post Graduate Institute of Medical Education and Research, Chandigarh, Nephrology, Chandigarh, India; ${ }^{3}$ Post Graduate Institute of Medical Education and Research, Chandigarh, Immunopathology, Chandigarh, India; ${ }^{4}$ Post Graduate Institute of Medical Education and Research, Chandigarh, Pulmonary Medicine, Chandigarh, India

Background: Tuberculosis is known to have diverse clinical presentations, some of which may mimic systemic autoimmune diseases like ANCA associated vasculitis and systemic lupus erythematous (SLE) ${ }^{1}$. Considering the paucity of specific biomarkers in rheumatologic practices, caution needs to be applied while interpreting ANA and ANCA results, especially in TB endemic areas. Previous studies on prevalence of autoantibodies in tuberculosis have shown contrasting results $2,3,4$

Objectives: To study the prevalence of ANCA subtypes and ANA in patients with bacteriologically confirmed pulmonary tuberculosis.

Methods: Patients with bacteriologically confirmed pulmonary tuberculosis were screened for recruitment. Anti-PR3, anti-MPO, anti-lactoferrin and anti-elastase ANCA subtypes were tested using enzyme-linked immunosorbent assay (ELISA). ANA was done by Indirect immunofluorescence (IIF) using Hep 2 cell lines. Patients who were positive for ANA were tested for presence for various extractable nuclear antigens using line immunoassay.

Results: Eighty nine patient were recruited in the study. Median age was 28 (range 20 - 46) years. The bacteriological confirmation was done via sputum examination in 81 (79 smear and 2 Gene Xpert) patients and bronchoalveolar lavage (BAL) fluid in 8 patients $(5$ smear and Gene Xpert). Out of 89,62 patients were treatment naïve for pulmonary TB. The clinical features were fever $(70 \%)$, Cough $(99 \%)$, expectoration $(99 \%)$, dyspnea( $25 \%)$, hemoptysis $(25 \%)$ and chest pain(13.5\%),. The radiological features were consolidation $(61 \%)$, reticulonodular opacities $(50 \%)$, cavity $(44 \%)$, bronchiectasis $(6 \%)$ and pleural effusion ( $8 \%)$.Among ANCA subtypes, anti-elastase was the most common and was positive in $86(96.62 \%)$ patients, followed by anti-PR3 seen in $7(7.8 \%)$ patients. No sera were found positive for anti-MPO and anti-lactoferrin antibodies. Six (6.7\%) patients had positive ANA (IIF).Line immunoassay in these patients was positive for SS-A/Ro-52 and U1-RNP in one patient each.

Conclusion: Anti PR3 ANCA positivity can be detected in patients with pulmonary tuberculosis and should be interpreted with caution in tuberculosis endemic areas. Anti-elastase ANCA was detected in majority of these patients and its role in differentiating tuberculosis from ANCA associated vasculitis needs further exploration.

\section{REFERENCES:}

[1] Al-Hadad I, Taha O. TB, or not TB: That is the question. Respir Med CME. 2010;3:192-4

[2] Lima I, Oliveira RC, Cabral MS, Atta A, Marchi S, Reis E, et al. Anti-PR3 and anti-MPO antibodies are not present in sera of patients with pulmonary tuberculosis. Rheumatol Int. 2014;34:1231-4

[3] Huan G, Yang G, Xiao-yu Q, Jiancheng X, Yan-qing S, Huan G, et al. Antineutrophil cytoplasmic antibodies in Chinese patients with tuberculosis. Rev Soc Bras Med Trop. 2018;51:475-8.
[4] Elkayam O, Caspi D, Lidgi M, Segal R. Auto-antibody profiles in patients with active pulmonary tuberculosis. Int J Tuberc Lung Dis. 2007;11:306-10

Disclosure of Interests: None declared

DOI: 10.1136/annrheumdis-2019-eular.4485

\section{FRI0650 A NAILFOLD CAPILLAROSCOPY STUDY IN A COHORT OF PATIENTS WITH ANTI-PHOSPHOLIPID ANTIBODIES}

Massimiliano Vasile ${ }^{1}$, Katia Stefanantoni ${ }^{1}$, Fulvia Ceccarelli ${ }^{1}$, Elena Marafioti ${ }^{1}$, Fabrizio Conti ${ }^{1}$, Valeria Riccieri ${ }^{1}$, Guido Valesini ${ }^{2}$. ${ }^{1}$ Sapienza Università di Roma, Medicina Interna e Specialità Mediche-Reumatologia, Roma, Italy; ${ }^{2}$ Sapienza Univesità di Roma, Medicina Interna e Specialità Mediche-Reumatologia, Roma, Italy

Background: Nailfold capillaroscopy (NC) is a simple and non-invasive diagnostic technique, able to investigate microvascular features. In subjects with anti-phospholipid autoantibodies (aPL) many different endothelial abnormalities have been described.

Objectives: We aimed to investigate the role of $\mathrm{NC}$ in aPL positive (aPL +) subjects, highlighting the main microvascular alterations, detected by $\mathrm{NC}$

Methods: We enrolled 39 patients with primary anti-phospholipids syndrome (pAPS) (32 females, mean age 43 years, mean disease duration 7 years), 47 patients with secondary anti-phospholipis syndrome (sAPS) due to Systemic Lupus Erythematosus (36 females, mean age 44 years, mean disease duration 15.5 years) and $17 \mathrm{aPL}+$ subjects without any autoimmune disease, defined as $\mathrm{aPL}$ carriers (16 females, mean age 42 years). All subjects underwent clinical and laboratory evaluations as well as NC with both characterization of morfological parameters and attribution of a semiquantitative score.

Results: The main NC findings were: morphological alterations (tortuous, bushy and/or ramified capillary) in $100 \%$ patients with pAPS, in $77 \%$ sAPS patients and in $88 \%$ aPL carriers; microhaemorragies in $56 \%$ pAPS patients, in $49 \%$ sAPS patients and $24 \%$ aPL carriers; enlarged hairpins in $23 \%, 41 \%$ and $18 \%$ subjects with pAPS, sAPS and aPL carriers respectively. In $6.3 \%$ sAPS patients, an early scleroderma pattern was detected.

A NC semiquantitative score 1 was found in $58.9 \%$ pAPS patients, in $57.4 \%$ sAPS patients and in $70.5 \%$ aPL carriers. Among those cases with abnormal NC findings, we found that a higher NC score (>2) was significantly more frequent in pAPS $(21.7 \%)$ and sAPS $(22.2 \%)$ patients respect to $\mathrm{aPL}$ carrier cases $(8.3 \%)(p<0,005)$.

We also found, among the main cardiovascular risk factors, that a higher score (1) was significantly associated with the presence of diabetes mellitus in pAPS patients $(\mathrm{p}<0,0001)$ and with arterial hypertension $(p<0,0004)$ and dyslipidemia $(p<0,0007)$ both in sAPS patients and aPL carriers.

Conclusion: Our findings show that some NC aspecific abnormalities are more frequently found in aPL positive subjects, mainly in pAPS and sAPS ones. Altough not specific, such NC features seem to be associated with the presence of vascular risk factors.

Thus NC could be regarded as a useful tool in order to evaluate microcirculation in aPL positive cases.

\section{REFERENCES:}

[1] Tavakol ME et al, Biomed Res Int 2015; 2015: 974530

[2] Maricq HR et al, Arthritis Rheum 1980; 23: 183 - 188.

[3] Vaz JLP et al, Rheumatology 2004;43:1025-7.

[4] Sulli A, J Rheum 2000;27:1574-6.

[5] Aslanidis S, Clin Exp Rheum 2011;29:307-9.

Disclosure of Interests: massimiliano vasile: None declared, Katia Stefanantoni Consultant for: Only 1 scientific advice for Italfarmaco in 2016, Fulvia Ceccarelli: None declared, Elena Marafioti: None declared, fabrizio conti: None declared, Valeria Riccieri: None declared, Guido Valesini: None declared

DOI: 10.1136/annrheumdis-2019-eular.8115 